\title{
Kaluza-Klein Dark Matter: Direct Detection vis-a-vis LHC
}

\section{Sebastian Arrenberg ${ }^{* a}$, Laura Baudis ${ }^{a}$, Kyoungchul Kong $^{b}$, Konstantin T. Matchev $^{c}$ and Jonghee Yoo $^{b}$}

${ }^{a}$ Physics Institute, University of Zürich

${ }^{b}$ Fermi National Accelerator Laboratory

${ }^{c}$ Physics Department, University of Florida

E-mails: arrenberg@physik.uzh.ch, laura.baudis@physik.uzh.ch, kckong@fnal.gov, matchev@phys.ufl.edu, yoo@fnal.gov

\begin{abstract}
We explore the phenomenology of Kaluza-Klein (KK) dark matter in very general models with universal extra dimensions (UEDs), emphasizing the complementarity between high-energy colliders and dark matter direct detection experiments. In models with relatively small mass splittings between the dark matter candidate and the rest of the (colored) spectrum, the collider sensitivity is diminished, but direct detection rates are enhanced. UEDs provide a natural framework for such mass degeneracies. We consider both 5-dimensional and 6-dimensional non-minimal UED models, and discuss the detection prospects for various KK dark matter candidates: the KK photon $\gamma_{1}(5 \mathrm{D})$ the KK Z-boson $Z_{1}(5 \mathrm{D})$ and the spinless KK photon $\gamma_{H}(6 \mathrm{D})$. We combine collider limits such as electroweak precision data and expected LHC reach, with cosmological constraints from WMAP and the sensitivity of current or planned direct detection experiments. Allowing for general mass splittings, we show that neither colliders, nor direct detection experiments by themselves can explore all of the relevant KK dark matter parameter space. Nevertheless, they probe different parameter space regions and the combination of the two types of constraints can be quite powerful. For example, in the case of $\gamma_{1}$ in 5D UEDs the relevant parameter space will be almost completely covered by the combined LHC and direct detection sensitivities expected in the near future. The work presented here is based on [1].
\end{abstract}

Identification of dark matter 2008

August 18-22, 2008

Stockholm, Sweden

${ }^{*}$ Speaker. 


\section{Introduction}

In the framework of UEDs [2] all standard model (SM) particles are promoted to one or more flat, compactified extra dimensions. An infinite number of new particles, called Kaluza-Klein tower, arises for every SM particle. Since at tree level their masses receive a dominant contribution $\sim \mathrm{TeV}$ arising from the momentum carried along the extra dimension(s) the spectrum is highly degenerated and thus radiative corrections are significant. The lightest Kaluza-Klein partner (LKP) is stable and if it is neutral, it can be a possible dark matter candidate. Considering the mass spectrum we did not restrict ourselves to the minimal UED (MUED) framework where vanishing boundary interactions at the cut-off scale are assumed, but rather allowed for a more general scenario taking the mass splitting

$$
\Delta_{q_{1}}=\frac{m_{q_{1}}-m_{L K P}}{m_{L K P}}
$$

between the LKP and the level one KK quarks as a free parameter.

\section{Relic density calculations}

Since a high degree of mass degeneracy occurs quite naturally in models with UEDs it is of particular importance to take coannihilations into account when computing the relic density of the LKP candidate [3]. The procedure is straightforward. After all heavier particles have decayed into it, the number density of the lightest species obeys a simple Boltzmann equation, where essentially the cross section has to be replaced by an effective cross section which depends on all annihilation cross sections and the mass splittings between the lightest particle and all other particles considered.

We computed the relic densities for $\gamma_{1}$ [3] and $Z_{1}$ in 5D UED taking coannihilations with all other level one KK particles into account. In the MUED framework the $\gamma_{1}$ is the LKP. The result of the computation is shown as a dotted, red line in Fig. 1(a). In a second approach a certain mass splitting $\Delta$ between the LKP and the KK quarks was assumed fixing the rest of the spectrum at their
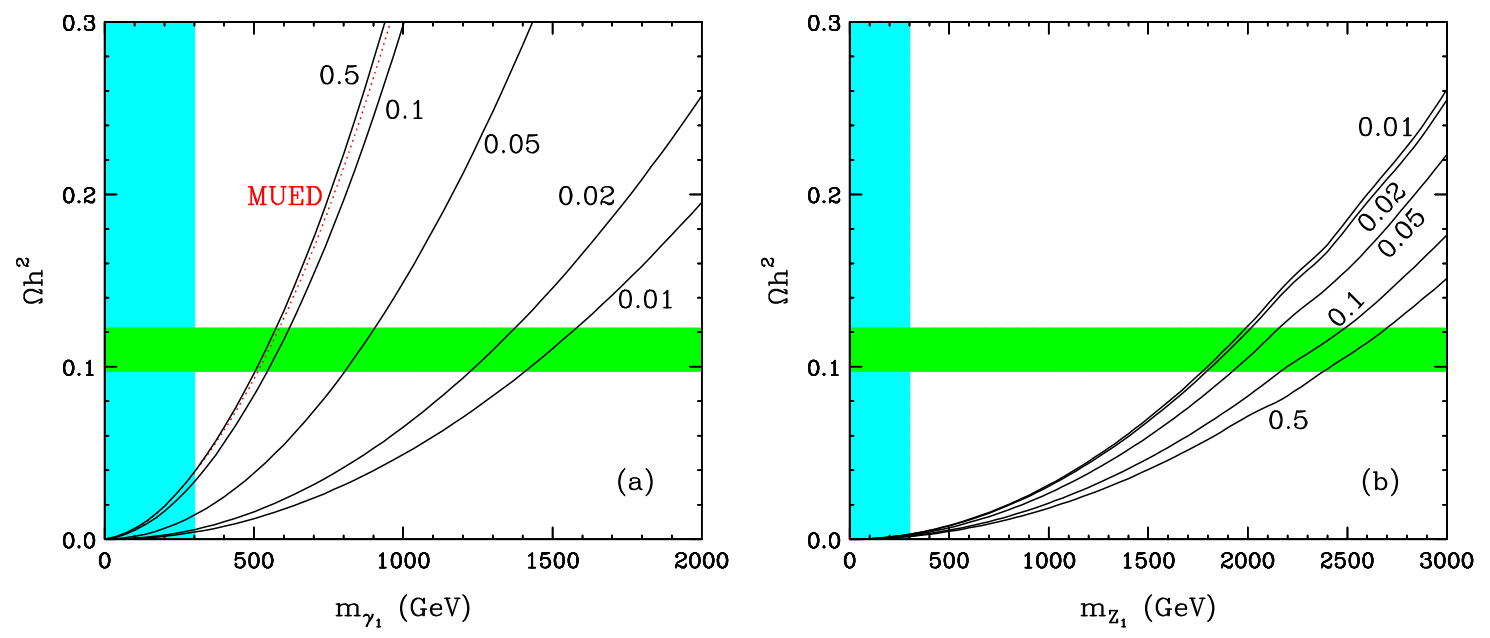

Figure 1: Relic density of the LKP and constraints from electroweak precision data and WMAP. 
MUED masses. Each line in the plot is labeled with the corresponding value of $\Delta$. Coannihilations thus decrease the predictions for the relic density. In the case of the $Z_{1}$ shown in Fig. 1(b) the quark masses were fixed in the same way as before. $Z_{1}$ and $W_{1}^{ \pm}$are assumed to be degenerate while the gluon is heavier than $Z_{1}$ by $10 \%$ and all other KK particles are heavier by $20 \%$. In this case the effect of coannihilations is inverted showing that its sign cannot easily be predicted. In both plots we also show constraints from electroweak precision data $[4,5]$ as a cyan vertical band and the preferred $2 \sigma$-WMAP region [6] as a green horizontal band. For example, a mass of $500 \mathrm{GeV}$ for the $\gamma_{1}$ LKP in 5D MUED is a good benchmark. ${ }^{1}$

\section{Direct LKP Detection - Predictions and Limits}

In order to explore constraints from direct detection experiments it is necessary to investigate the elastic scattering between the dark matter particle and target nuclei. For all three LKP candidates considered here, $\gamma_{1}$ and $Z_{1}$ in 5D UED and $\gamma_{H}$ in 6D UED, there are two Feynman diagrams arising from KK quark exchange and one from Higgs boson exchange, contributing to the scattering cross section at tree level [1]. Here we only consider spin-independent scattering.

The theoretical predictions are shown in Fig. 2. We fixed the Higgs mass at $120 \mathrm{GeV}$ and assumed $\Delta$ to be between $1 \%$ which is the upper boundary of the respective shaded area and $50 \%$ which is the lower boundary. The plot also contains cross section limits from CDMS [7] and XENON10 [8] as well as expected sensitivities from future experiments. Current experiments already exclude small mass splittings while future experiments should cover most of the relevant parameter space.

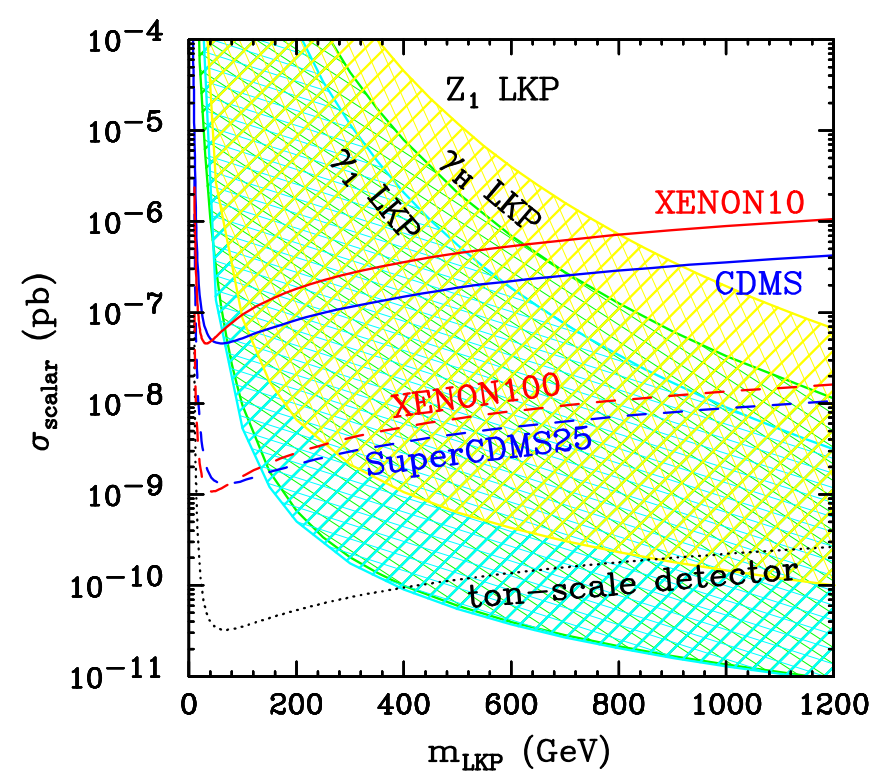

Figure 2: Theoretical predictions for spin-independent LKP-nucleon cross sections and sensitivities from current and planned future direct detection experiments.

\footnotetext{
${ }^{1}$ Note that the relic density including coannihilations of $\gamma_{H}$ in 6D UED has not been investigated yet.
} 


\section{Limits on Kaluza-Klein Dark Matter}

In addition to calculating the limits on the spin-independent cross sections we also performed a more detailed analysis of the LKP specific parameter space.

For example, one can translate the information from Fig. 2 into the $\Delta$ vs. WIMP mass plane when fixing the Higgs mass. This is shown in Fig. 3 for $\gamma_{1}$ and $Z_{1}$ in 5D UED. All mass splittings below the respective limit curve are excluded. As expected direct detection experiments are particularly sensitive to small $\Delta$ 's. Additionally both plots contain relic density constraints providing upper limits on the LKP mass. The black solid line accounts for all the dark matter in the universe while the two black dotted lines show the bounds assuming that the LKP would contribute only $1 \%$ or $10 \%$ to the total amount of dark matter. The green shaded region represents the preferred $2 \sigma$-WMAP region [6]. For the case of the $\gamma_{1}$ a collider study of the $4 \ell+B_{T}$ channel yielded the result that with a luminosity of $100 \mathrm{fb}^{-1}$ the yellow shaded region should be covered by the LHC [9]. The LHC is thus more sensitive to large mass splittings. All three probes are highly complementary and in the case of the $\gamma_{1}$ the entire parameter space could be covered by ton-scale direct detection experiments.

On the other hand, one can also interchange the role of the mass splitting and the Higgs mass. In Fig. 4 we show limits on the Higgs mass fixing $\Delta$ at $10 \%$. The excluded regions are below and to the left of each limit curve. The asymptotic behaviour is related to the decoupling of the Higgs exchange for large Higgs masses. In each plot the horizontal black line represents the current Higgs mass bound of $114 \mathrm{GeV}$ while the diagonal line shows the limit from oblique corrections [5]. ${ }^{2}$ From the observation that the Higgs mass dependence in direct detection experiments only shows up in already excluded parts of the parameter space it can be concluded that it plays a secondary role interpreting those experiments. Even future direct detection experiments will only probe a small part of the relevant parameter space, however the LHC will be more sensitive with respect to the Higgs mass.
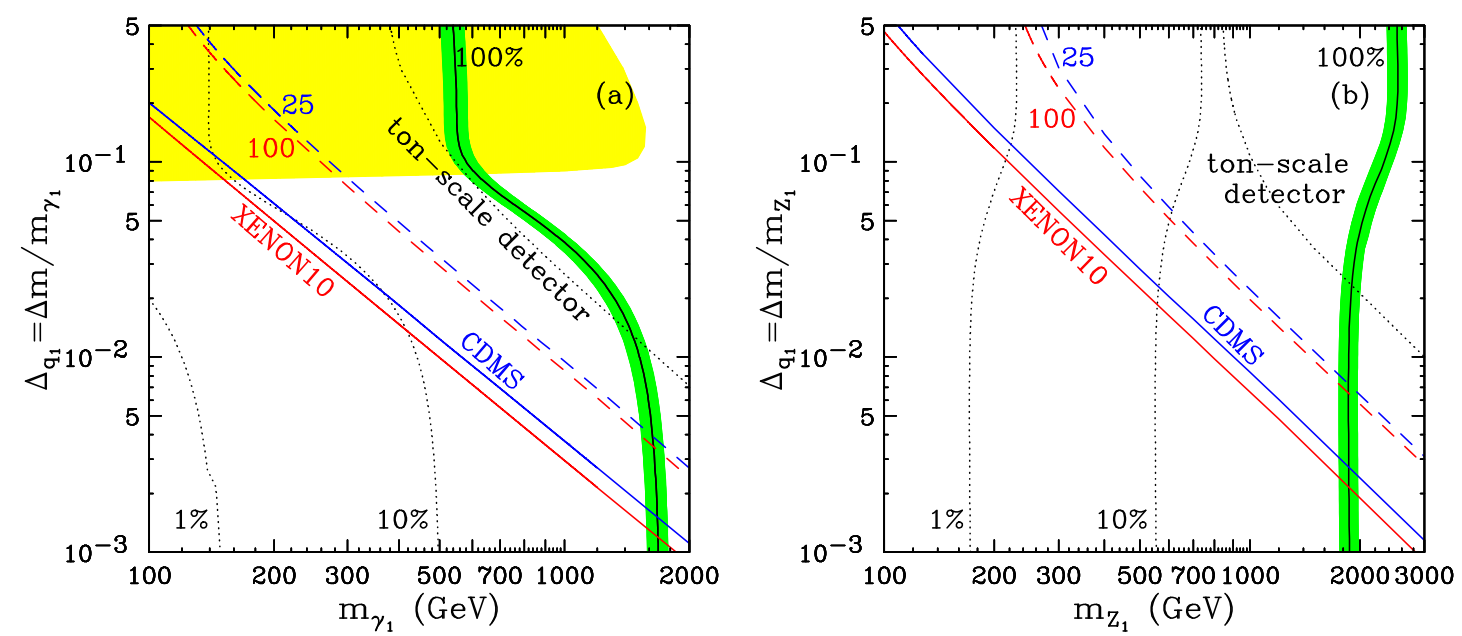

Figure 3: Limits from direct detection experiments, LHC studies and WMAP in the $\Delta$ vs. $m_{L K P}$ plane.

\footnotetext{
${ }^{2}$ Note that these corrections were computed considering the $\gamma_{1}$ to be the LKP in MUED. Thus the corresponding bound in Fig. 4(b) has been included only for illustrative reasons.
} 

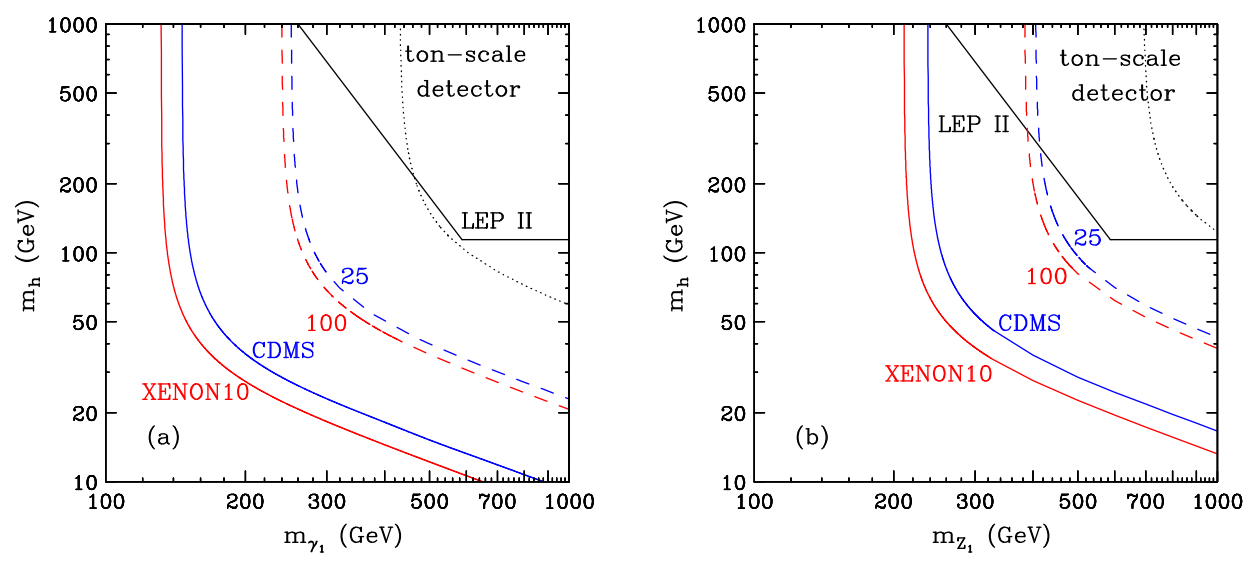

Figure 4: Limits from direct detection experiments and collider studies in the $m_{h}$ vs. $m_{L K P}$ plane.

For a more detailed analysis, including the parameter space of $\gamma_{H}$ LKP in 6D UED and spindependent interactions, we refer to [1].

\section{Conclusion}

We performed a comprehensive phenomenological analysis of Kaluza-Klein dark matter, including constraints from direct detection experiments, collider studies and cosmology considering 5D and 6D UED. It was shown that all three approaches are highly complementary and combining them substantially diminishes the relevant parameter space. Direct detection experiments restrict small values of $\Delta$ whereas colliders are sensitive to large mass splittings. Cosmology on the other hand rules out large LKP masses and as shown here these two parameters ( $\Delta$ and $m_{L K P}$ ) are the relevant quantities in analyzing UEDs. Moreover the importance of coannihilations for relic density computations should be emphasized.

Important investigations for the future in the context of UEDs would be further LHC and more detailed relic density studies for certain LKP candidates such as the $\gamma_{H}$ LKP in 6D UED.

\section{References}

[1] S. Arrenberg, L. Baudis, K. Kong, K.T. Matchev and J. Yoo, Phys. Rev. D 78, 056002 (2008) [hep-ph/0805.4210]

[2] D. Hooper and S. Profumo, Phys. Rept. 453, 29 (2007) [hep-ph/0701197]

[3] K. Kong and K.T. Matchev, JHEP 0601, 038 (2006) [hep-ph / 0509119 ]

[4] T. Appelquist and H. U. Yee, Phys. Rev. D 67, 055002 (2003) [hep-ph / 0211023 ]

[5] I. Gogoladze and C. Macesanu, Phys. Rev. D 74, 093012 (2006) [hep-ph / 0605207 ]

[6] J. Dunkley et al. (WMAP Collaboration), [astro-ph/0803.0586]

[7] Z. Ahmed et al. (CDMS Collaboration), [astro-ph/0802 . 3530]

[8] J. Angle et al. (XENON10 Collaboration), Phys. Rev. Lett. 100, 021303 (2008) [astro-ph/0706.0039]

[9] H. C. Cheng, K.T. Matchev and M. Schmaltz, Phys. Rev. D 66, 056006 (2002) [hep-ph/ 0205314$]$ 